\title{
Inequalities for sums of adapted random fields in Banach spaces and their application to strong law of large numbers
}

\author{
Hung Thang Dang, Cong Son Ta and Manh Cuong Tran*
}

"Correspondence:

cuongtm@vnu.edu.vn

Department of Mathematics, VNU

Hanoi University of Science, 334

NguyenTrai St., Hanoi, Vietnam

\begin{abstract}
We obtain inequalities for sums of adapted random fields in $p$-uniformly smooth Banach spaces, and we extend Kolmogorov and Marcinkiewicz-Zygmund strong laws for blockwise $\alpha$-martingale difference fields.

MSC: 60B11;60B12; 60F15; 60G42

Keywords: adapted random field; blockwise $\alpha$-martingale difference field; Marcinkiewicz-Zygmund strong law of larger numbers; $p$-uniformly smooth Banach space
\end{abstract}

\section{Introduction}

The concept of blockwise-dependence was introduced by Móricz [1]. Móricz's [1] and Gaposhkin [2] showed that some properties of sequences of independent random variables can be applied to sequences consisting of independent blocks. Huan et al. [3] extended the strong laws of large numbers to blockwise-martingale difference arrays in Banach spaces. Recently, Móricz et al. [4] introduced the concept of blockwise $M$-dependence for a double array of random variables and established a version of the Kolmogorov SLLN for double arrays of random variables which are blockwise $M$-dependent. The results of Móricz and Stadtmüller and Thalmaier [5] were generalized by Stadtmüller and Thanh [6].

The aim of this paper is to investigate inequalities for sums of random fields and the strong law of large numbers of arbitrary random fields taking values in a Banach space. In Section 2, we introduce $\alpha$-strong adapted random fields, $\alpha$-strong* adapted random fields, blockwise $\alpha$-martingale difference fields and prove some useful lemmas. In Section 3, inequalities for sums of $\alpha$-strong adapted random fields and $\alpha$-strong* adapted random fields in $p$-uniformly smooth Banach spaces are given. Section 4 contains the main results including the SLLN for a such blockwise $\alpha$-martingale difference field taking values in a $p$-uniformly smooth Banach space, in which the results of $[3,6,7]$ will be generalized.

Throughout this paper, the symbol $C$ will denote a generic constant $(0<C<\infty)$ which is not necessarily the same one in each appearance.

\section{Preliminaries and some useful lemmas}

Let $\mathbb{E}$ be a real separable Banach space. $\mathbb{E}$ is said to be $p$-uniformly smooth $(1 \leq p \leq 2)$ if there exists a finite positive constant $C$ such that for all $\mathbb{E}$-valued martingales $\left\{S_{n} ; 1 \leq n \leq\right.$

\section{算 Springer}

○2014 Dang et al.; licensee Springer. This is an Open Access article distributed under the terms of the Creative Commons Attribution License (http://creativecommons.org/licenses/by/2.0), which permits unrestricted use, distribution, and reproduction in any medium, provided the original work is properly cited. 
$m\}$

$$
E\left\|S_{m}\right\|^{p} \leq C \sum_{n=1}^{m} E\left\|S_{n}-S_{n-1}\right\|^{p} .
$$

Clearly, every real separable Banach space is 1-uniformly smooth and the real line (the same as any Hilbert space) is 2-uniformly smooth. If a real separable Banach space is $p$-uniformly smooth for some $1<p \leq 2$ then it is $r$-uniformly smooth for all $r \in[1, p)$.

Let $d$ be a positive integer, the set of all integer $d$-dimensional lattice points will be denoted by $\mathbb{Z}^{d}$ and the set of all positive integer $d$-dimensional lattice points will be denoted by $\mathbb{N}^{d}$. For $\mathbf{m}=\left(m_{1}, \ldots, m_{d}\right) \in \mathbb{Z}^{d}, \mathbf{n}=\left(n_{1}, \ldots, n_{d}\right) \in \mathbb{Z}^{d}, \boldsymbol{\alpha}=\left(\alpha_{1}, \ldots, \alpha_{d}\right) \in \mathbb{R}^{d}$ denote $[\mathbf{m}, \mathbf{n})=\prod_{i=1}^{d}\left[m_{i}, n_{i}\right)$ is a $d$-dimensional rectangle, $\mathbf{m}+\mathbf{n}=\left(m_{1}+n_{1}, \ldots, m_{d}+n_{d}\right)$, $\mathbf{m}-\mathbf{n}=\left(m_{1}-n_{1}, \ldots, m_{d}-n_{d}\right), \mathbf{2}^{\mathbf{n}}=\left(2^{n_{1}}, \ldots, 2^{n_{d}}\right),\left|\mathbf{n}^{\alpha}\right|=\prod_{i=1}^{d} n_{i}^{\alpha_{i}}, \mathbf{1}=(1, \ldots, 1) \in \mathbb{N}^{d}$. We write $\mathbf{m} \preceq \mathbf{n}$ (or $\mathbf{n} \succeq \mathbf{m}$ ) if $m_{i} \leq n_{i}, 1 \leq i \leq d ; \mathbf{m} \prec \mathbf{n}$ if $\mathbf{m} \preceq \mathbf{n}$, and $\mathbf{m} \neq \mathbf{n}$. For $x \geq 0$, let [ $\left.x\right]$ denote the greatest integer less than or equal to $x$, we use $\log ^{+} x$ to denote the $\log (x \vee 1)$ (the logarithms are to base 2).

For nondecreasing sequences of positive integers $\left\{\alpha_{i}(k), k \geq 1\right\}(1 \leq i \leq d)$, for $\mathbf{n} \in \mathbb{N}^{d}$, let $\alpha(\mathbf{n})=\left(\alpha_{1}\left(n_{1}\right), \ldots, \alpha_{d}\left(n_{d}\right)\right)$.

Let $(\Omega, \mathcal{F}, P)$ be a probability space, $\mathbb{E}$ be a real separable Banach space, and $\mathcal{B}(\mathbb{E})$ be the $\sigma$-algebra of all Borel sets in $\mathbb{E}$. Let $\left\{X_{\mathbf{k}}, \mathbf{n} \preceq \mathbf{k} \preceq \mathbf{N}\right\}$ be a field of $\mathbb{E}$-valued random variables and $\left\{\mathcal{F}_{\mathbf{k}}, \mathbf{n} \preceq \mathbf{k} \preceq \mathbf{N}\right\}$ be a field of nondecreasing sub- $\sigma$-algebras of $\mathcal{F}$ with respect to the partial order $\preceq$ on $\mathbb{N}^{d}$ such that $X_{\mathbf{k}}$ is $\mathcal{F}_{\mathbf{k}}$-measurable for all $\mathbf{n} \preceq \mathbf{k} \preceq \mathbf{N}$, then $\left\{X_{\mathbf{k}}, \mathcal{F}_{\mathbf{k}}, \mathbf{n} \preceq \mathbf{k} \preceq \mathbf{N}\right\}$ is said to be an adapted field.

Let $\left\{X_{\mathbf{k}}, \mathcal{F}_{\mathbf{k}}, \mathbf{n} \preceq \mathbf{k} \preceq \mathbf{N}\right\}$ be an adapted field, we adopt the convention that $\mathcal{F}_{\mathbf{k}}=\{\emptyset, \Omega\}$ if $\mathbf{k} \nsucceq \mathbf{n}$. For $\mathbf{k} \in \mathbb{Z}^{d}(\mathbf{k} \preceq \mathbf{N}-\mathbf{1})$ set

$$
\mathcal{F}_{\mathbf{k}}^{i}=\sigma\left\{\mathcal{F}_{\mathbf{l}}: \mathbf{l}=\left(l_{1}, \ldots, l_{d}\right), l_{j} \leq k_{j}(j \neq i) \text { and } l_{i}=k_{i}\right\}
$$

for all $1 \leq i \leq d$, and

$$
\mathcal{F}_{\mathbf{k}}^{*}=\sigma\left\{\mathcal{F}_{\mathbf{k}}^{(i)}: 1 \leq i \leq d\right\}
$$

The adapted field $\left\{X_{\mathbf{k}}, \mathcal{F}_{\mathbf{k}}, \mathbf{n} \preceq \mathbf{k} \preceq \mathbf{N}\right\}$ is said to be $\alpha$-strong adapted (or strong adapted) if $E\left(X_{\mathbf{k}} \mid \mathcal{F}_{\mathbf{l}-\alpha(\mathbf{l})}^{*}\right)$ (or $\left.E\left(X_{\mathbf{k}} \mid \mathcal{F}_{\mathbf{l}-\mathbf{1}}^{*}\right)\right)$ is $\mathcal{F}_{\mathbf{k}}^{i}$-measurable for all $\mathbf{n} \preceq \mathbf{l} \preceq \mathbf{k} \preceq \mathbf{N}$, and $1 \leq i \leq d$.

The adapted field $\left\{X_{\mathbf{k}}, \mathcal{F}_{\mathbf{k}}, \mathbf{n} \preceq \mathbf{k} \preceq \mathbf{N}\right\}$ is said to be $\alpha$-strong* adapted (or strong* adapted) if $\left\{X_{\mathbf{k}} I_{A}, \mathcal{F}_{\mathbf{k}}, \mathbf{n} \preceq \mathbf{k} \preceq \mathbf{N}\right\}$ is $\alpha$-strong adapted (or strong adapted) for all $A \in \sigma\left(X_{\mathbf{k}}\right)$.

Example 2.1 Let $\left\{X_{n}, \mathcal{G}_{n}: 1 \leq n \leq N\right\}$ be an adapted sequence of $\mathbb{E}$-valued random variables and set

$$
\begin{array}{ll}
X_{\mathbf{k}}=X_{k} & \text { if } \mathbf{k}=(k, k, \ldots, k) \quad \text { and } \quad X_{\mathbf{k}}=0 \quad \text { if } \mathbf{k} \neq(k, k, \ldots, k) \\
\mathcal{G}_{\mathbf{k}}=\mathcal{G}_{k} & \text { if } \mathbf{k}=(k, k, \ldots, k) \quad \text { and } \quad \mathcal{G}_{\mathbf{k}}=\{\emptyset, \Omega\} \quad \text { if } \mathbf{k} \neq(k, k, \ldots, k) .
\end{array}
$$

Let $\mathcal{F}_{\mathbf{k}}=\sigma\left\{\mathcal{G}_{\mathbf{l}}, \mathbf{l} \preceq \mathbf{k}\right\}$ for all $\mathbf{k} \succeq \mathbf{1}$, then $E\left(X_{\mathbf{k}} I_{A} \mid \mathcal{F}_{\mathbf{k}-\mathbf{1}}^{*}\right)=E\left(X_{k} I_{A} \mid \mathcal{G}_{k-1}\right) \in \mathcal{G}_{k}=\mathcal{F}_{\mathbf{k}}^{i}$ if $\mathbf{k}=$ $(k, k, \ldots, k), E\left(X_{\mathbf{k}} I_{A} \mid \mathcal{F}_{\mathbf{k}-\mathbf{1}}^{*}\right)=0$ if otherwise, for all $A \in \sigma\left(X_{\mathbf{k}}\right)$ and $\mathbf{1} \preceq \mathbf{k} \preceq \mathbf{N}=(N, \ldots, N)$, so $\left\{X_{\mathbf{k}}, \mathcal{F}_{\mathbf{k}}: \mathbf{1} \preceq \mathbf{k} \preceq \mathbf{N}\right\}$ is a strong* adapted field. 
Example 2.2 Let $\left\{Y_{\mathbf{k}}, \mathbf{n} \preceq \mathbf{k} \preceq \mathbf{N}\right\}$ be a field of independent random variables. Put $\mathcal{F}_{\mathbf{k}}=$ $\sigma\left(Y_{\mathbf{i}}, \mathbf{i} \preceq \mathbf{k}\right)$ and $X_{\mathbf{k}}=\prod_{\mathbf{i} \leq \mathbf{k}} Y_{\mathbf{i}}$, so $\left\{X_{\mathbf{k}}, \mathbf{n} \preceq \mathbf{k} \preceq \mathbf{N}\right\}$ is not a field of independent random variables. If $E\left|X_{\mathbf{k}}\right|<\infty$ for all $\mathbf{n} \preceq \mathbf{k} \preceq \mathbf{N}$, then $\left\{X_{\mathbf{k}}, \mathcal{F}_{\mathbf{k}}, \mathbf{n} \preceq \mathbf{k} \preceq \mathbf{N}\right\}$ is a strong* adapted field.

The adapted field $\left\{X_{\mathbf{k}}, \mathcal{F}_{\mathbf{k}}, \mathbf{n} \preceq \mathbf{k} \preceq \mathbf{N}\right\}$ is said to be an $\alpha$-martingale difference field if $E\left(X_{\mathbf{k}} \mid \mathcal{F}_{\mathbf{k}-\alpha(\mathbf{k})}^{i}\right)=0$ for all $\mathbf{n} \preceq \mathbf{k} \preceq \mathbf{N}$ and $1 \leq i \leq d$.

When $\alpha(\mathbf{k})=\left(M_{1}, \ldots, M_{d}\right)=\mathbf{M}$ for all $\mathbf{n} \preceq \mathbf{k} \preceq \mathbf{N}$ then the adapted field $\left\{X_{\mathbf{k}}, \mathcal{F}_{\mathbf{k}}, \mathbf{n} \preceq \mathbf{k} \preceq\right.$ $\mathbf{N}$ \} is said to be an $\mathbf{M}$-martingale difference field.

When $\alpha(\mathbf{k})=1$ for all $\mathbf{n} \preceq \mathbf{k} \preceq \mathbf{N}$ then the field $\left\{X_{\mathbf{k}}, \mathcal{F}_{\mathbf{k}}, \mathbf{n} \preceq \mathbf{k} \preceq \mathbf{N}\right\}$ is a martingale difference field which was introduced by Huan et al. [3] in case $d=2$.

\section{Remark 2.3}

- Let $\left\{X_{\mathbf{k}}, \mathcal{F}_{\mathbf{k}}: \mathbf{n} \preceq \mathbf{k} \preceq \mathbf{N}\right\}$ be a field of martingale differences, then it is strong adapted, but it is not necessarily a strong* adapted field.

- Let $\left\{X_{\mathbf{k}}, \mathbf{k} \in \mathbb{Z}^{d}\right\}$ be a field of $m$-dependence random variables with mean 0 . Put $\mathcal{F}_{\mathbf{k}}=\sigma\left(X_{\mathbf{l}}, \mathbf{l} \preceq \mathbf{k}\right)$ and $\mathbf{M}=(m, \ldots, m)$, then $E\left(X_{\mathbf{k}} \mid \mathcal{F}_{\mathbf{k}-\mathbf{M}}^{i}\right)=E X_{\mathbf{k}}=0$ for all $\mathbf{k} \in \mathbb{Z}^{d}$, $1 \leq i \leq d$. Therefore, $\left\{X_{\mathbf{k}}, \mathcal{F}_{\mathbf{k}}, \mathbf{k} \in \mathbb{Z}^{d}\right\}$ is a field of $\mathbf{M}$-martingale differences.

Example 2.4 Let $\left\{X_{\mathbf{k}}, \mathbf{k} \in \mathbb{Z}^{d}\right\}$ be a field of independent random elements with mean 0 . Put $\mathcal{F}_{\mathbf{k}}=\sigma\left(X_{\mathbf{l}}, \mathbf{l} \preceq \mathbf{k}\right)$, then $E\left(X_{\mathbf{k}} \mid \mathcal{F}_{\mathbf{k}-\mathbf{1}}^{i}\right)=0$ for all $\mathbf{k} \in \mathbb{Z}^{d}, 1 \leq i \leq d$. Therefore, $\left\{X_{\mathbf{k}}, \mathcal{F}_{\mathbf{k}}, \mathbf{k} \in \mathbb{Z}^{d}\right\}$ is a field of martingale differences and a strong* adapted field.

Set $Y_{\mathbf{k}}=\sum_{\mathbf{k}+\mathbf{1}-\alpha(\mathbf{k}) \leq \mathbf{l} \leq \mathbf{k}} X_{\mathbf{l}}$, then $\left\{Y_{\mathbf{k}}, \mathcal{F}_{\mathbf{k}}, \mathbf{k} \in \mathbb{Z}^{d}\right\}$ is a field of $\alpha$-martingale differences.

Example 2.5 Let $\left\{Y_{\mathbf{k}}, \mathbf{k} \in \mathbb{N}^{d}\right\}$ be a field of independent random variables with mean 0 . Put $\mathcal{F}_{\mathbf{k}}=\sigma\left(Y_{\mathbf{l}}, \mathbf{l} \preceq \mathbf{k}\right)$ and $X_{\mathbf{k}}=\prod_{\mathbf{l} \leq \mathbf{k}} Y_{\mathbf{l}}$, so $\left\{X_{\mathbf{k}}, \mathbf{k} \in \mathbb{N}^{d}\right\}$ is not a field of independent random variables. If $E\left|X_{\mathbf{k}}\right|<\infty$ for all $\mathbf{k} \succeq \mathbf{1}$, then $E\left(X_{\mathbf{k}} \mid \mathcal{F}_{\mathbf{k}-1}^{i}\right)=0, E\left(X_{\mathbf{k}} I_{A} \mid \mathcal{F}_{\mathbf{k}-1}^{*}\right)=E\left(X_{\mathbf{k}} I_{A}\right) \in \mathcal{F}_{\mathbf{k}}^{i}$ for all $A \in \sigma\left(X_{\mathbf{k}}\right), \mathbf{k} \geq \mathbf{1}, 1 \leq i \leq d$. Therefore, $\left\{X_{\mathbf{k}}, \mathcal{F}_{\mathbf{k}}, \mathbf{k} \in \mathbb{N}^{d}\right\}$ is a field of martingale differences and a strong* adapted field.

For strictly increasing sequence of positive integers $\left\{\omega_{i}(k), k \geq 1\right\}$, with $\omega_{i}(1)=1(1 \leq i \leq$ $d$ ), and $\mathbf{k} \in \mathbb{N}^{d}$, we set

$$
\omega(\mathbf{k})=\left(\omega_{1}\left(k_{1}\right), \ldots, \omega_{d}\left(k_{d}\right)\right), \quad \Delta_{\mathbf{k}}=[\omega(\mathbf{k}), \omega(\mathbf{k}+\mathbf{1})) .
$$

The adapted field $\left\{X_{\mathbf{k}}, \mathcal{F}_{\mathbf{k}}, \mathbf{k} \in \mathbb{N}^{d}\right\}$ is said to be a blockwise-adapted field (respectively, blockwise- $\alpha$-strong adapted, blockwise- $\alpha$-strong* adapted, blockwise- $\alpha$-martingale difference field, blockwise-M-martingale difference field, blockwise-martingale difference field) with respect to the blocks $\left\{\Delta_{\mathbf{k}}, \mathbf{k} \in \mathbb{N}^{d}\right\}$ if for each $\mathbf{k} \in \mathbb{N}^{d},\left\{X_{\mathbf{k}}, \mathcal{F}_{\mathbf{k}}, \mathbf{k} \in \Delta_{\mathbf{k}}\right\}$ is an adapted field (respectively, $\alpha$-strong adapted, $\alpha$-strong* adapted, $\alpha$-martingale difference field, $\mathbf{M}$-martingale difference field, martingale difference field).

Example 2.6 Let $X_{\mathbf{k}}, \mathcal{F}_{\mathbf{k}}, Y_{\mathbf{k}}$ as in Example 2.4. Set $Z_{\mathbf{i}}=Y_{\mathbf{i}-2^{\mathbf{k}}+\mathbf{1}}$ and $\mathcal{G}_{\mathbf{i}}=\sigma\left(Z_{\mathbf{u}} ; 2^{\mathbf{k}} \preceq \mathbf{u} \preceq \mathbf{i}\right)$, $2^{\mathbf{k}} \preceq \mathbf{i} \prec \mathbf{2}^{\mathbf{k}+\mathbf{1}}, \mathbf{k} \succeq \mathbf{0}$ then $\left\{Z_{\mathbf{n}}, \mathcal{G}_{\mathbf{n}} ; \mathbf{n} \succeq \mathbf{1}\right\}$ is a blockwise- $\alpha$-martingale differences and a blockwise- $\alpha$-strong* adapted field with respect to the blocks $\prod_{k=1}^{d}\left[2^{k}, 2^{k+1}\right)$.

To prove the main result we need the following lemmas. 
Lemma 2.7 Let $\mathbb{E}$ be a real separable p-uniformly smooth Banach space for some $1 \leq$ $p \leq 2$. Then there exists a positive constant $C$ such that all strong adapted random fields $\left\{X_{\mathbf{k}}, \mathcal{F}_{\mathbf{k}}, \mathbf{n} \preceq \mathbf{k} \preceq \mathbf{N}\right\}$ in $\mathbb{E}$ we have

$$
E\left[\max _{\mathbf{n} \leq \mathbf{k} \leq \mathbf{N}}\left\|\sum_{\mathbf{n} \leq \mathbf{i} \leq \mathbf{k}}\left(X_{\mathbf{i}}-E\left(X_{\mathbf{i}} \mid \mathcal{F}_{\mathbf{i}-\mathbf{1}}^{*}\right)\right)\right\|^{p}\right] \leq C \sum_{\mathbf{n} \leq \mathbf{k} \leq \mathbf{N}} E\left\|X_{\mathbf{k}}\right\|^{p} .
$$

Proof We set

$$
S_{\mathbf{k}}=\sum_{\mathbf{1} \leq \mathbf{i} \leq \mathbf{k}}\left(X_{\mathbf{i}}-E\left(X_{\mathbf{i}} \mid \mathcal{F}_{\mathbf{i}-\mathbf{1}}^{*}\right)\right) .
$$

Firstly, for $d=1$, note that $\left\{\max _{1 \leq i \leq k}\left\|S_{i}\right\|, \mathcal{F}_{k}: n \leq k \leq N\right\}$ is a nonnegative sub-martingale. Applying Doob's inequality and by (2.1), we have (2.2). We assume that (2.2) holds for $d-1$, we wish to show that it holds for $d$.

Denote $\mathbf{k}=\left(\mathbf{k}^{\prime}, k_{d}\right) ; \mathbf{k}=\left(\mathbf{n}^{\prime}, n_{d}\right) ; \mathbf{N}=\left(\mathbf{N}^{\prime}, N_{d}\right) ;$ with $\mathbf{k}^{\prime}, \mathbf{n}^{\prime}, \mathbf{N}^{\prime} \in \mathbb{N}^{d-1}$; set

$$
Y_{k_{d}}=\max _{\mathbf{n}^{\prime} \leq \mathbf{k}^{\prime} \leq \mathbf{N}^{\prime}}\left\|S_{\left(\mathbf{k}^{\prime} ; k_{d}\right)}\right\|
$$

for each $n_{d} \leq k_{d} \leq N_{d}$, we have

$$
\begin{aligned}
E\left(S_{\left(\mathbf{k}^{\prime} ; k_{d}\right)} \mid \mathcal{F}_{\left(\mathbf{k}^{\prime} ; k_{d}-1\right)}^{d}\right)= & E\left(S_{\left(\mathbf{k}^{\prime} ; k_{d}-1\right)} \mid \mathcal{F}_{\left(\mathbf{k}^{\prime} ; k_{d}-1\right)}^{d}\right) \\
& +\sum_{\mathbf{n}^{\prime} \leq \mathbf{k}^{\prime} \leq \mathbf{N}^{\prime}}\left(E\left(X_{\left(\mathbf{k}^{\prime} ; k_{d}\right)}-E\left(X_{\left(\mathbf{k}^{\prime} ; k_{d}\right)} \mid \mathcal{F}_{\left(\mathbf{k}^{\prime}-\mathbf{1}, k_{d}-1\right)}^{*}\right) \mid \mathcal{F}_{\left(\mathbf{k}^{\prime} ; k_{d}-1\right)}^{d}\right)\right) \\
= & S_{\left(\mathbf{k}^{\prime} ; k_{d}-1\right)}
\end{aligned}
$$

that means that for each $\mathbf{n}^{\prime} \leq \mathbf{k}^{\prime} \leq \mathbf{N}^{\prime}$ then $\left\{S_{\left(\mathbf{k}^{\prime} ; k_{d}\right)} ; \mathcal{F}_{\left(\mathbf{k}^{\prime} ; k_{d}\right)}^{d}: n_{d} \leq k_{d} \leq N_{d}\right\}$, we find that $\left\{Y_{k_{d}} ; \mathcal{F}_{\left(\mathbf{k}^{\prime} ; k_{d}\right)}: n_{d} \leq k_{d} \leq N_{d}\right\}$ is a nonnegative sub-martingale sequence. Applying Doob's inequality, we obtain

$$
E \max _{\mathbf{n} \leq\left(\mathbf{k}^{\prime}, k_{d}\right) \leq \mathbf{N}}\left\|S_{\left(\mathbf{k}^{\prime} ; k_{d}\right)}\right\|^{p}=E \max _{n_{d} \leq k_{d} \leq N_{d}} Y_{k_{d}}^{p} \leq C \cdot E Y_{N_{d}}^{p}=C \cdot E \max _{\mathbf{n}^{\prime} \leq \mathbf{k}^{\prime} \leq \mathbf{N}^{\prime}}\left\|S_{\left(\mathbf{k}^{\prime} ; N_{d}\right)}\right\|^{p} .
$$

Set

$$
X_{\mathbf{k}^{\prime}}^{d-1}=\sum_{n_{d} \leq k_{d} \leq N_{d}} X_{\left(\mathbf{k}^{\prime} ; k_{d}\right)} ; \quad \mathcal{F}_{\mathbf{k}^{\prime}}^{d-1}=\sigma\left(\mathcal{F}_{\left(\mathbf{k}^{\prime} ; k_{d}\right)}^{d-1}: n_{d} \leq k_{d} \leq N_{d}\right) .
$$

We note that $\mathcal{F}_{\left(\mathbf{k}^{\prime}, k_{d}\right)}^{i}=\left(\mathcal{F}_{\mathbf{k}^{\prime}}^{d-1}\right)^{i}, \mathcal{F}_{\left(\mathbf{k}^{\prime}, k_{d}\right)}^{*}=\left(\mathcal{F}_{\mathbf{k}^{\prime}}^{d-1}\right)^{*}$, for all $n_{d} \leq k_{d} \leq N_{d}, 1 \leq i \leq d$, then $\left\{X_{\mathbf{k}^{\prime}}^{d-1} ; \mathcal{F}_{\mathbf{k}^{\prime}}^{d-1}: \mathbf{n}^{\prime} \leq \mathbf{k}^{\prime} \leq \mathbf{N}^{\prime}\right\}$ is a strong adapted field. Therefore, by the inductive assumption and inequality (2.1) we have

$$
\begin{aligned}
E\left[\max _{\mathbf{n} \leq\left(\mathbf{k}^{\prime}, k_{d}\right) \leq \mathbf{N}}\left\|S_{\left(\mathbf{k}^{\prime} ; k_{d}\right)}\right\|^{p}\right] & \leq C \cdot \sum_{\mathbf{n}^{\prime} \leq \mathbf{k}^{\prime} \leq \mathbf{N}^{\prime}} E\left\|_{n_{d} \leq k_{d} \leq N_{d}} X_{\left(\mathbf{k}^{\prime} ; k_{d}\right)}-E\left(X_{\left(\mathbf{k}^{\prime} ; k_{d}\right)} \mid \mathcal{F}_{\left(\mathbf{k}^{\prime}-\mathbf{1}, k_{d}-1\right)}^{*}\right)\right\|^{p} \\
& \leq C \sum_{\mathbf{1} \leq \mathbf{k} \leq \mathbf{n}} E\left\|X_{\mathbf{k}}-E\left(X_{\mathbf{k}} \mid \mathcal{F}_{\mathbf{k}-\mathbf{1}}^{*}\right)\right\|^{p} \leq C \sum_{\mathbf{1} \leq \mathbf{k} \leq \mathbf{n}} E\left\|X_{\mathbf{k}}\right\|^{p} .
\end{aligned}
$$


Remark 2.8 If $\left\{X_{\mathbf{k}} ; \mathbf{k} \succeq \mathbf{1}\right\}$ is an $\mathbb{E}$-valued martingale difference field, from Lemma 2.7, we obtain Lemma 2.4 in [3] for $d=2$ and Corollary 3.2 in [8] for $d \geq 2$ (with $p=q$ ).

We note that if $\left\{X_{\mathbf{k}}, \mathcal{F}_{\mathbf{k}}: \mathbf{n} \preceq \mathbf{k} \preceq \mathbf{N}\right\}$ is strong adapted, when $d=1$ then $\left\{X_{k}-E\left(X_{k} \mid \mathcal{F}_{k-1}^{*}\right)\right.$, $\left.\mathcal{F}_{k}: n \leq k \leq N\right\}$ is a sequences of martingale differences, but when $d>1$ then $\left\{X_{\mathbf{k}}-\right.$ $\left.E\left(X_{\mathbf{k}} \mid \mathcal{F}_{\mathbf{k}-1}^{*}\right), \mathcal{F}_{\mathbf{k}}: \mathbf{n} \preceq \mathbf{k} \preceq \mathbf{N}\right\}$ is not necessarily a field of martingale differences, because $X_{\mathbf{k}}-E\left(X_{\mathbf{k}} \mid \mathcal{F}_{\mathbf{k}-1}^{*}\right)$ may not be $\mathcal{F}_{\mathbf{k}}$-measurable (see [9], Example 3.1).

Lemma 2.9 Let $1<p \leq 2, \alpha=\left(\alpha_{1}, \ldots, \alpha_{d}\right)$ where $\alpha_{1}, \ldots, \alpha_{d}$ are positive constants, $1=\alpha_{1}=$ $\cdots=\alpha_{q}<\alpha_{q+1} \leq \cdots \leq \alpha_{d}$ and $X$ be a random variable taking values in a real separable Banach space $\mathbb{E}$. Then there exists a positive constant $C$ such that

(i) $\sum_{\mathbf{n} \geq \mathbf{1}} \frac{1}{\left|\mathbf{n}^{\alpha}\right|^{p}} \int_{0}^{\left|\mathbf{n}^{\alpha}\right| p} P\left\{\|X\|^{p} \geq t\right\} d t \leq C \cdot\left(E\|X\| \log _{+}^{q-1}\|X\|+1\right)$,

(ii) $\sum_{\mathbf{n} \geq \mathbf{1}} \frac{1}{\left|\mathbf{n}^{\alpha}\right|} \int_{\left|\mathbf{n}^{\alpha}\right|}^{\infty} P\{\|X\| \geq t\} d t \leq C \cdot E\|X\| \log _{+}^{q}\|X\|$.

Proof Denote $d(k)=\sum_{n_{1} \cdots n_{q}=k} 1$, by Lemma 3.1 of Gut [10] we have

$$
\sum_{j=k}^{\infty} \frac{d(j)}{j^{p}} \sim C \frac{(\log k)^{q-1}}{k^{p-1}} .
$$

Hence, we have

$$
\begin{aligned}
& \sum_{\mathbf{n}} \frac{1}{\left|\mathbf{n}^{\alpha}\right|^{p}} \int_{0}^{\left|\mathbf{n}^{\alpha}\right|^{p}} P\left\{\|X\|^{p} \geq t\right\} d t \\
& \leq \sum_{\mathbf{n}} \frac{1}{\left|\mathbf{n}^{\alpha}\right|^{p}}+\sum_{\mathbf{n}} \frac{1}{\left|\mathbf{n}^{\alpha}\right|^{p}} \int_{1}^{\left|\mathbf{n}^{\alpha}\right|^{p}} P\left\{\|X\|^{p} \geq t\right\} d t \\
& \leq C+C \sum_{\mathbf{n}} \frac{1}{\left|\mathbf{n}^{\alpha}\right|^{p}} \int_{1}^{\left|\mathbf{n}^{\alpha}\right|^{p}} P\left\{\|X\|^{p} \geq t\right\} d t \\
& \leq C+C \sum_{k, n_{q+1}, \ldots, n_{d}=1}^{\infty} d(k) \frac{1}{k^{p} \cdot n_{q+1}^{p \alpha_{q+1}} \cdots n_{d}^{p \alpha_{d}}} \sum_{j=1}^{\left[k n_{q+1}^{\left.\alpha_{q+1} \ldots n_{d} \alpha_{d}\right]}\right.} E\left(\|X\|^{p} I(j \leq\|X\|<j+1)\right)
\end{aligned}
$$

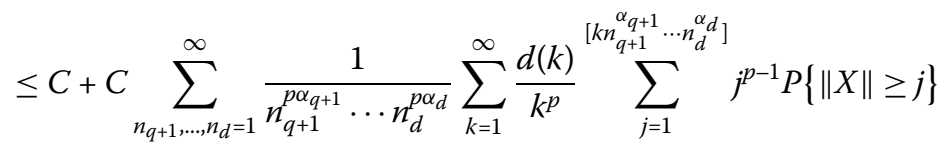

$$
\begin{aligned}
& \leq C+C \sum_{n_{q+1}, \ldots, n_{d}=1}^{\infty} \frac{1}{n_{q+1}^{p \alpha_{q+1}} \cdots n_{d}^{p \alpha_{d}}} \sum_{j=1}^{\left[n_{q_{+1} \cdots n_{d}}^{\left.\alpha_{q+1} \ldots \alpha_{d}\right]}\right.} j^{p-1} P\{\|X\| \geq j\} \sum_{k=1}^{\infty} \frac{d(k)}{k^{p}} \\
& +C \sum_{n_{q+1}, \ldots, n_{d}=1}^{\infty} \frac{1}{n_{q+1}^{p \alpha_{q+1} \cdots n_{d}^{p \alpha_{d}}}} \sum_{j=\left[n_{q+1}^{\left.\alpha_{q+1} \ldots n_{d}^{\alpha}\right]+1}\right.}^{\infty} j^{p-1} P\{\|X\| \geq j\} \sum_{k=\left[j / n_{q+1}^{\left.\alpha_{q+1} \ldots n_{d}^{\alpha}\right]}\right.}^{\infty} \frac{d(k)}{k^{p}} \\
& \leq C+C \sum_{n_{q+1}, \ldots, n_{d}=1}^{\infty} \frac{1}{n_{q+1}^{\alpha_{q+1}} \cdots n_{d}^{\alpha_{d}}} \sum_{j=1}^{\infty}(\log i)^{q-1} P\{\|X\| \geq j\} \leq C+C E\|X\| \log _{+}^{q-1}\|X\| .
\end{aligned}
$$


Now we prove (ii). We have by Lemma 3 of Stadtmüller and Thalmaier [5]

$$
g(j)=\sum_{\substack{\alpha_{q+1} \ldots n_{d}^{\alpha} \leq j \\ 1 \leq n_{1} \cdots n_{q} \cdot n_{q+1} \cdots n_{d}}} 1 \sim C \frac{j(\log j)^{q-1}}{(q-1) !} \text { as } j \rightarrow \infty .
$$

Denote $\Delta g(j)=g(j)-g(j-1)$, we get

$$
\begin{aligned}
& \sum_{\mathbf{n} \succeq \mathbf{1}} \frac{1}{\left|\mathbf{n}^{\alpha}\right|} \int_{\left|\mathbf{n}^{\alpha}\right|}^{\infty} P\{\|X\| \geq t\} d t \\
& \quad=\sum_{k=1}^{\infty} \frac{1}{k} \Delta g(k) \int_{k}^{\infty} P\{\|X\| \geq t\} d t=\sum_{k=1}^{\infty} \frac{1}{k} \Delta g(k) \sum_{i=k}^{\infty} \int_{i}^{i+1} P\{\|X\| \geq t\} d t \\
& \leq \sum_{k=1}^{\infty} \frac{1}{k} \Delta g(k) \sum_{j=k}^{\infty} j P\{j \leq\|X\|<j+1\}=\sum_{j=1}^{\infty} j P\{j \leq\|X\|<j+1\} \sum_{k=1} \frac{1}{k} \Delta g(k) \\
& \leq C \sum_{j=1}^{\infty} j P\{j \leq\|X\|<j+1\} \sum_{k=1}^{\infty}\left(\frac{1}{k}-\frac{1}{k+1}\right) k(\log k)^{q-1} \\
& \quad+C \sum_{j=1}^{\infty} P\{j \leq\|X\|<j+1\} j(\log j)^{q-1} \\
& \leq C \sum_{j=1}^{\infty} j P\{j \leq\|X\|<j+1\}(\log j)^{q-1} \sum_{k=1}^{j} \frac{1}{k} \\
& \quad+C \sum_{j=1}^{\infty} P\{j \leq\|X\|<j+1\} j(\log j)^{q-1} \\
& \leq C \sum_{j=1}^{\infty} P\{j \leq\|X\|<j+1\} j(\log j)^{q} \leq C E\|X\| \log _{+}^{q}\|X\| . \\
& \quad
\end{aligned}
$$

Lemma 2.10 Let $\left\{a_{\mathbf{n}}, \mathbf{n} \succeq \mathbf{1}\right\}$ be a nondecreasing field of positive constants such that

$$
1<\liminf _{\mathbf{n} \prec \mathbf{m} \leq \mathbf{n}+\mathbf{1}} \frac{a_{\mathbf{m}}}{a_{\mathbf{n}}} \leq \limsup _{\mathbf{n} \prec \mathbf{m} \leq \mathbf{n}+\mathbf{1}} \frac{a_{\mathbf{m}}}{a_{\mathbf{n}}} \leq M .
$$

If $\left\{x_{\mathbf{n}}, \mathbf{n} \succeq \mathbf{1}\right\}$ is a field of constants and

$$
\lim _{|\mathbf{n}| \rightarrow \infty} x_{\mathbf{n}}=0
$$

then

$$
\lim _{|\mathbf{n}| \rightarrow \infty} \frac{1}{a_{\mathbf{n}}} \sup _{\mathbf{k} \leq \mathbf{n}}\left|\sum_{\mathbf{1} \leq \mathbf{j} \leq \mathbf{k}} a_{\mathbf{j}+\mathbf{1}} x_{\mathbf{j}}\right|=0 .
$$

Proof First, we prove that

$$
\frac{1}{a_{\mathbf{n}}} \sum_{\mathbf{1} \leq \mathbf{j} \leq \mathbf{n}} a_{\mathbf{j}+\mathbf{1}} \leq C
$$


By (2.3), there exist a constant $0<\delta<1$ and $\mathbf{n}_{0}$ such that for all $\mathbf{m} \succ \mathbf{n} \succeq \mathbf{n}_{0}$ then $\frac{a_{\mathbf{n}}}{a_{\mathbf{m}}} \leq \delta$. We have for all $\mathbf{n} \succeq \mathbf{1}$

$$
\frac{1}{a_{\mathbf{n}}} \sum_{\mathbf{1} \leq \mathbf{j} \leq \mathbf{n}} a_{\mathbf{j}+\mathbf{1}} \leq M \frac{1}{a_{\mathbf{n}+\mathbf{1}}} \sum_{\mathbf{1} \leq \mathbf{j} \leq \mathbf{n}} a_{\mathbf{j}+\mathbf{1}} \leq M\left(\left|\mathbf{n}_{0}\right|+\frac{1}{(1-\delta)^{d}}\right)
$$

and we have (2.4).

For every $\epsilon>0$, there exists $N>0$ such that for all $|\mathbf{n}| \geq N,\left|x_{\mathbf{n}}\right| \leq \frac{\epsilon}{C}$ so that

$$
\frac{1}{a_{\mathbf{n}}} \sup _{\mathbf{k} \leq \mathbf{n}}\left|\sum_{\mathbf{1} \leq \mathbf{j} \leq \mathbf{k}} a_{\mathbf{j}+\mathbf{1}} x_{\mathbf{j}}\right| \leq \frac{1}{a_{\mathbf{n}}} \sup _{|\mathbf{k}|<\mathbf{N}}\left|\sum_{|\mathbf{j}| \leq \mathbf{k}} a_{\mathbf{j}+\mathbf{1}} x_{\mathbf{j}}\right|+\epsilon .
$$

The conclusion of the lemma follows upon letting $|\mathbf{n}| \rightarrow \infty$ and then $\epsilon \rightarrow 0$.

\section{Inequalities for sums of adapted random fields}

The first theorem characterizes the $p$-uniformly smooth Banach spaces.

Theorem 3.1 Let $1 \leq p \leq 2$ and $\mathbb{E}$ be a separable Banach space, then the following three statements are equivalent:

(i) $\mathbb{E}$ is p-uniformly smooth.

(ii) There exists a positive constant $C$ such that for all $\alpha$-strong adapted random fields $\left\{X_{\mathbf{k}}, \mathcal{F}_{\mathbf{k}} ; \mathbf{n} \preceq \mathbf{k} \preceq \mathbf{m}\right\}$ in $\mathbb{E}$ we have

$$
\begin{gathered}
E\left[\max _{\mathbf{n} \leq \mathbf{k} \leq \mathbf{m}}\left\|\sum_{\mathbf{n} \leq \mathbf{i} \leq \mathbf{k}} X_{\mathbf{i}}-E\left(X_{\mathbf{i}} \mid \mathcal{F}_{\mathbf{i}-\alpha(\mathbf{i})}^{*}\right)\right\|^{p}\right] \\
\leq C\left|\alpha^{p-1}(\mathbf{m})\right| \sum_{\mathbf{n} \leq \mathbf{k} \leq \mathbf{m}} E\left\|X_{\mathbf{k}}\right\|^{p} .
\end{gathered}
$$

Proof We first prove the implication ((i) $\Rightarrow$ (ii)). If $\mathbf{m}-\mathbf{n} \preceq \alpha(\mathbf{m})$, (3.1) is trivial.

If $\mathbf{m}-\mathbf{n} \succeq \alpha(\mathbf{m})$, note that if $\{\alpha(\mathbf{k}), \mathbf{k} \succeq \mathbf{1}\}$ is a nondecreasing field of positive, for all $\mathbf{n} \preceq \mathbf{i} \preceq \alpha(\mathbf{m})+\mathbf{n}-\mathbf{1}$ then

$$
\left\{X_{\mathbf{i}+(\mathbf{u}-\mathbf{1}) \alpha(\mathbf{m}),} \mathcal{F}_{\mathbf{i}+\mathbf{u} \alpha(\mathbf{m})+\alpha(\mathbf{i}+\mathbf{u} \alpha(\mathbf{m}))} ; \mathbf{n} \preceq \mathbf{i}+(\mathbf{u}-\mathbf{1}) \alpha(\mathbf{m}) \preceq \mathbf{m}\right\}
$$

are $\alpha$-strong adapted fields. Set

$$
A_{\mathbf{i}}=\{\mathbf{k} ; 0 \preceq \mathbf{k} \alpha(\mathbf{m})+\mathbf{i} \preceq \mathbf{m}-\mathbf{n}\} \quad \text { and } \quad Y_{\mathbf{k}}=X_{\mathbf{k}}-E\left(X_{\mathbf{k}} \mid \mathcal{F}_{\mathbf{k}-\alpha(\mathbf{k})}^{*}\right) .
$$

Then we have

$$
\begin{aligned}
& E\left(\max _{\mathbf{n} \leq \mathbf{k} \leq \mathbf{m}}\left\|\sum_{\mathbf{n} \leq \mathbf{i} \leq \mathbf{k}} X_{\mathbf{i}}-E\left(X_{\mathbf{i}} \mid \mathcal{F}_{\mathbf{i}-\alpha(\mathbf{i})}^{*}\right)\right\|^{p}\right) \\
& \quad \leq E\left(\sum_{\mathbf{0} \leq \mathbf{i} \leq \alpha(\mathbf{m})-\mathbf{1}} \max _{\mathbf{k} \in A_{\mathbf{i}}}\left\|\sum_{\mathbf{0} \leq \mathbf{u} \leq \mathbf{k}} Y_{\mathbf{u} \alpha(\mathbf{m})+\mathbf{i}+\mathbf{n}}\right\|\right)^{p} \\
& \quad \leq \alpha^{p-1}(\mathbf{m})\left(\sum_{\mathbf{0} \leq \mathbf{i} \leq \alpha(\mathbf{m})-\mathbf{1}} E \max _{\mathbf{k} \in A_{\mathbf{i}}}\left\|\sum_{\mathbf{0} \leq \mathbf{u} \leq \mathbf{k}} Y_{\mathbf{u} \alpha(\mathbf{m})+\mathbf{i}+\mathbf{n}}\right\|^{p}\right)
\end{aligned}
$$




$$
\begin{aligned}
& \leq C \alpha^{p-1}(\mathbf{m}) \sum_{\mathbf{0} \leq \mathbf{i} \leq \alpha(\mathbf{m})-\mathbf{1}}\left(\sum_{\mathbf{k} \in A_{\mathbf{i}}} E\left\|Y_{\mathbf{k} \alpha(\mathbf{m})+\mathbf{i}+\mathbf{n}}\right\|^{p}\right) \\
& =C \alpha^{p-1}(\mathbf{m}) \sum_{\mathbf{n} \leq \mathbf{i} \leq \mathbf{m}} E\left\|X_{\mathbf{i}}\right\|^{p} \quad \text { by }(2.2),
\end{aligned}
$$

again establishing (3.1). If $\bigvee_{i=1}^{d}\left(m_{i}-n_{i}<\alpha_{i}\left(m_{i}\right)\right)$, the proof is similar to (3.2).

$(($ ii $) \Rightarrow(\mathrm{i}))$ Let $\left\{X_{n}, \mathcal{G}_{n}, n \geq 1\right\}$ be a martingale difference sequence in $\mathbb{E}$.

For $\mathbf{n}^{\prime} \succ \mathbf{1}, n_{d} \geq 1$, put

$$
\begin{aligned}
& X_{\left(\mathbf{n}^{\prime} ; n_{d}\right)}=X_{n_{d}} \quad \text { if } \mathbf{n}^{\prime}=\mathbf{1} \quad \text { and } \quad X_{\left(\mathbf{n}^{\prime} ; n_{d}\right)}=0 \quad \text { if } \mathbf{n}^{\prime} \succ \mathbf{1}, \\
& \mathcal{F}_{\left(\mathbf{n}^{\prime} ; n_{d}\right)}=\mathcal{G}_{\mathbf{n}^{\prime}} .
\end{aligned}
$$

Then $\left\{X_{\mathbf{n}}, \mathcal{F}_{\mathbf{n}}, \mathbf{n} \succeq \mathbf{1}\right\}$ is an $\alpha$-strong adapted field with $\alpha(\mathbf{n})=1$ by (ii); we have (2.1) and then $\mathbb{E}$ is $p$-uniformly smooth.

From now on, let $\left\{\Gamma_{i}(k), k \geq 1\right\}$ be strictly increasing sequences of positive integers with $\Gamma_{i}(1)=1(1 \leq i \leq d)$. For $\mathbf{m} \in \mathbb{N}^{d}, \mathbf{n} \in \mathbb{N}^{* d}$, we introduce the following notations:

$$
\begin{aligned}
& \Gamma(\mathbf{n})=\left(\Gamma_{1}\left(n_{1}\right), \ldots, \Gamma_{d}\left(n_{d}\right)\right), \quad \Delta^{\mathbf{m}}=[\Gamma(\mathbf{m}), \Gamma(\mathbf{m}+\mathbf{1})), \quad \Delta_{\mathbf{n}}^{\mathbf{m}}=\Delta_{\mathbf{n}} \cap \Delta^{\mathbf{m}}, \\
& I_{\mathbf{m}}=\left\{\mathbf{n}: \Delta_{\mathbf{n}}^{\mathbf{m}} \neq \emptyset\right\}, \quad c_{\mathbf{m}}=\operatorname{card} I_{\mathbf{m}}, \\
& \varphi_{1}(\mathbf{n})=\sum_{\mathbf{k} \succeq \mathbf{1}}^{\infty} c_{\mathbf{k}} I_{\Delta^{\mathbf{k}}}(\mathbf{n}), \quad \phi_{1}(\mathbf{n})=\max _{\mathbf{k} \leq \mathbf{n}} \varphi_{1}(\mathbf{k}), \\
& \varphi_{2}(\mathbf{n})=\sum_{\mathbf{k} \succeq \mathbf{1}}|\alpha(\Gamma(\mathbf{k}+\mathbf{1}))| \cdot c_{\mathbf{k}} I_{\Delta^{\mathbf{k}}}(\mathbf{n}), \quad \phi_{2}(\mathbf{n})=\max _{\mathbf{k} \leq \mathbf{n}} \varphi_{2}(\mathbf{k}),
\end{aligned}
$$

where $I_{\Delta^{(\mathbf{k})}}$ denotes the indicator function of the set $\Delta^{(\mathbf{k})} ; \mathbf{k} \in \mathbb{N}^{d}$.

Let $\left\{X_{\mathbf{n}}, \mathcal{F}_{\mathbf{n}} ; \mathbf{n} \in \mathbb{N}^{d}\right\}$ be a blockwise- $\alpha$-adapted field taking values in the Banach space $\mathbb{E}$ with respect to the blocks $\left\{\Delta_{\mathbf{n}} ; \mathbf{n} \in \mathbb{N}^{d}\right\}$, we put

$$
T_{\mathbf{n}}=\sup _{\mathbf{k} \in \Delta^{\mathbf{n}}}\left\|\sum_{\Gamma(\mathbf{n}) \preceq \mathbf{i} \leq \mathbf{k}} X_{\mathbf{i}}-E\left(X_{\mathbf{i}} \mid \mathcal{F}_{\mathbf{i}-\alpha(\mathbf{i})}^{*}\right)\right\| .
$$

Theorem 3.2 Let $\mathbb{E}$ be a p-uniformly smooth Banach space $(1 \leq p \leq 2)$. Then there exists a positive constant $C$ such that for all strong blockwise- $\alpha$-strong adapted random fields $\left\{X_{\mathbf{n}}, \mathcal{F}_{\mathbf{n}} ; \mathbf{n} \succeq \mathbf{1}\right\}$ in $\mathbb{E}$ with respect to the blocks $\left\{\Delta_{\mathbf{k}}, \mathbf{k} \succeq \mathbf{1}\right\}$ and every nondecreasing field of positive constants $\left\{a_{\mathbf{n}}, \mathbf{n} \succeq \mathbf{1}\right\}$ we have

$$
\sum_{\mathbf{n} \succeq \mathbf{1}} \frac{1}{a_{\Gamma(\mathbf{n}+\mathbf{1})}^{p} \varphi_{2}^{p-1}(\Gamma(\mathbf{n}))} \mathbb{E} T_{\mathbf{n}}^{p} \leq C \sum_{\mathbf{n} \succeq \mathbf{1}} \frac{1}{a_{\mathbf{n}}^{p}} E\left\|X_{\mathbf{n}}\right\|^{p} .
$$

Proof For $\mathbf{m} \in \mathbb{N}^{d}, \mathbf{n} \in I_{\mathbf{m}}$, we set

$$
\begin{aligned}
& r_{\mathbf{n}}^{\mathbf{m}}(i)=\min \left\{j: j \in\left[\omega_{i}\left(n_{i}\right), \omega_{i}\left(n_{i}+1\right)\right) \cap\left[\Gamma_{i}\left(m_{i}\right), \Gamma_{i}\left(m_{i}+1\right)\right)\right\}, \\
& r_{\mathbf{n}}^{\mathbf{m}}=\left(r_{\mathbf{n}}^{\mathbf{m}}(1), \ldots, r_{\mathbf{n}}^{\mathbf{m}}(d)\right), \quad T_{\mathbf{n}}^{\mathbf{m}}=\max _{u \in \Delta_{\mathbf{n}}^{\mathbf{m}}}\left\|\sum_{r_{\mathbf{n}}^{\mathbf{m}} \leq \mathbf{i} \leq \mathbf{u}} X_{\mathbf{i}}\right\| .
\end{aligned}
$$


We have $T_{\mathbf{m}} \leq \sum_{\mathbf{n} \in I_{\mathbf{m}}} T_{\mathbf{m}}^{\mathbf{n}}$ for $\mathbf{m} \in \mathbb{N}^{d}$. Applying the $C_{r}$ inequality, we have

$$
\begin{aligned}
\frac{E\left(T_{\mathbf{m}}\right)^{p}}{a_{\Gamma(\mathbf{m}+\mathbf{1})}^{p} \varphi_{2}^{p-1}(\Gamma(\mathbf{m}))} & \leq \frac{c_{\mathbf{m}}^{p-1}}{a_{\Gamma(\mathbf{m}+\mathbf{1})}^{p} \varphi_{2}(\Gamma(\mathbf{m}))^{p-1}} \sum_{\mathbf{n} \in I_{\mathbf{m}}} E\left(T_{\mathbf{n}}^{\mathbf{m}}\right)^{p} \\
& \leq \frac{c_{\mathbf{n}}^{p-1} \cdot C \cdot\left|\alpha^{p-1}(\Gamma(\mathbf{m}+\mathbf{1}))\right|}{\left(a_{\Gamma(\mathbf{m}+\mathbf{1})}\right){ }^{p}\left(\varphi_{2}(\Gamma(\mathbf{m}))\right)^{p-1}} \sum_{\mathbf{k} \in I_{\mathbf{m}}} \sum_{\mathbf{i} \in \Delta_{\mathbf{n}}^{\mathbf{m}}} E\left\|X_{\mathbf{i}}\right\|^{p} \quad \text { (by Lemma 2.7) } \\
& =\frac{C}{a_{\Gamma(\mathbf{m}+\mathbf{1})}^{p}} \sum_{\mathbf{i} \in \Delta^{\mathbf{m}}} E\left\|X_{\mathbf{i}}\right\|^{p} \leq C \sum_{\mathbf{i} \in \Delta^{\mathbf{m}}} \frac{1}{a_{i}^{p}} E\left\|X_{\mathbf{i}}\right\|^{p}
\end{aligned}
$$

When $\alpha(\mathbf{i})=\mathbf{M}$ for all $\mathbf{i} \succeq \mathbf{1}$, with a note that $\varphi_{2}(\mathbf{m})=|\mathbf{M}| \varphi_{1}(\mathbf{m})$ for all $\mathbf{m} \succeq \mathbf{1}$, we have the following corollary.

Corollary 3.3 Let $\mathbb{E}$ be a p-uniformly smooth Banach space $(1 \leq p \leq 2)$. Then there exists a positive constant $C$ such that for all strong blockwise-M-adapted random fields $\left\{X_{\mathbf{n}}, \mathcal{F}_{\mathbf{n}}\right.$ : $\mathbf{n} \succeq \mathbf{1}\}$ in $\mathbb{E}$ with respect to the blocks $\left\{\Delta_{\mathbf{k}}, \mathbf{k} \succeq \mathbf{1}\right\}$ and every nondecreasing field of positive constants $\left\{a_{\mathbf{n}}, \mathbf{n} \succeq \mathbf{1}\right\}$ we have

$$
\sum_{\mathbf{n} \geq \mathbf{1}} \frac{1}{a_{\Gamma(\mathbf{n}+\mathbf{1})}^{p} \varphi_{1}^{p-1}(\Gamma(\mathbf{n}))} \mathbb{E} T_{\mathbf{n}}^{p} \leq C \sum_{\mathbf{n} \geq \mathbf{1}} \frac{1}{a_{\mathbf{n}}^{p}} E\left\|X_{\mathbf{n}}\right\|^{p} .
$$

Theorem 3.4 $\mathbb{E}$ is a p-uniformly smooth Banach space $(1 \leq p \leq 2),\left\{X_{\mathbf{n}}, \mathcal{F}_{\mathbf{n}}, \mathbf{n} \succeq \mathbf{1}\right\}$ is a blockwise $\alpha$-strong*-adapted random field in $\mathbb{E}$ with respect to the blocks $\left\{\Delta_{\mathbf{k}}, \mathbf{k} \succeq \mathbf{1}\right\}$. $\left\{\psi_{\mathbf{n}}(x) \in \mathbb{R}^{+}\right\}$is a field of a positive Borel function which has the following property:

$$
C_{\mathbf{n}} \frac{u^{\lambda_{\mathbf{n}}}}{v^{\lambda_{\mathbf{n}}}} \leq \frac{\psi_{\mathbf{n}}(u)}{\psi_{\mathbf{n}}(v)} \leq D_{\mathbf{n}} \frac{u^{\mu_{\mathbf{n}}}}{v^{\mu_{\mathbf{n}}}} \quad \text { for all } u \geq v>0,
$$

where $C_{\mathbf{n}} \geq 1, D_{\mathbf{n}} \geq 1, \lambda_{\mathbf{n}} \geq 1,0<\mu_{\mathbf{n}} \leq p .\left\{a_{\mathbf{n}}, \mathbf{n} \geq \mathbf{1}\right\}$ is a nondecreasing field of positive constants satisfying (2.3). Then there exists a positive constant $C$ such that for all $\epsilon>0$, we have

$$
\sum_{\mathbf{n} \geq \mathbf{1}} P\left(T_{\mathbf{n}} \geq \epsilon a_{\Gamma(\mathbf{n}+\mathbf{1})} \varphi_{2}^{(p-1) / p}(\Gamma(\mathbf{n}))\right) \leq C \sum_{\mathbf{n} \succeq \mathbf{1}} A_{\mathbf{n}} \frac{E \psi_{\mathbf{n}}\left(\left\|X_{\mathbf{n}}\right\|\right)}{\psi_{\mathbf{n}}\left(a_{\mathbf{n}}\right)},
$$

where $A_{\mathbf{n}}=\max \left\{\frac{1}{C_{\mathbf{n}}}, D_{\mathbf{n}}\right\}$.

Proof For each $\mathbf{n} \succeq \mathbf{1}$, set

$$
\begin{aligned}
& Y_{\mathbf{n}}=X_{\mathbf{n}} I\left(\left\|X_{\mathbf{n}}\right\| \leq a_{\mathbf{n}}\right), \quad Z_{\mathbf{n}}=X_{\mathbf{n}} I\left(\left\|X_{\mathbf{n}}\right\|>a_{\mathbf{n}}\right), \\
& U_{\mathbf{n}}=\sup _{\mathbf{k} \in \Delta^{\mathbf{n}}}\left\|\sum_{\Gamma(\mathbf{n}) \leq \mathbf{i} \leq \mathbf{k}} Y_{\mathbf{i}}-E\left(Y_{\mathbf{i}} \mid \mathcal{F}_{\mathbf{i}-\alpha(\mathbf{i})}^{*}\right)\right\|, \quad V_{\mathbf{n}}=\sup _{\mathbf{k} \in \Delta^{\mathbf{n}}}\left\|\sum_{\Gamma(\mathbf{n}) \leq \mathbf{i} \leq \mathbf{k}} Z_{\mathbf{i}}-E\left(Z_{\mathbf{i}} \mid \mathcal{F}_{\mathbf{i}-\alpha(\mathbf{i})}^{*}\right)\right\| .
\end{aligned}
$$

Since $\left\{X_{\mathbf{n}}, \mathcal{F}_{\mathbf{n}}, \mathbf{n} \succeq \mathbf{1}\right\}$ is a blockwise- $\alpha$-strong* adapted field with respect to the blocks $\left\{\Delta_{\mathbf{m}}, \mathbf{m} \succeq \mathbf{1}\right\}$, it is clear that $\left\{Y_{\mathbf{n}}, \mathcal{F}_{\mathbf{n}}, \mathbf{n} \succeq \mathbf{1}\right\}$ and $\left\{Z_{\mathbf{n}}, \mathcal{F}_{\mathbf{n}}, \mathbf{n} \succeq \mathbf{1}\right\}$ are blockwise $\alpha$-strong adapted fields with respect to the blocks $\left\{\Delta_{\mathbf{m}}, \mathbf{m} \succeq \mathbf{1}\right\}$. Moreover, for $\mathbf{n} \in \mathbb{N}^{d}$,

$$
X_{\mathbf{n}}+E\left(X_{\mathbf{n}} \mid \mathcal{F}_{\mathbf{n}-\alpha(\mathbf{n})}\right)=\left(Y_{\mathbf{n}}+E\left(Y_{\mathbf{n}} \mid \mathcal{F}_{\mathbf{n}-\alpha(\mathbf{n})}\right)\right)+\left(Z_{\mathbf{n}}+E\left(Z_{\mathbf{n}} \mid \mathcal{F}_{\mathbf{n}-\alpha(\mathbf{n})}\right)\right) .
$$


Then $T_{\mathbf{n}} \leq U_{\mathbf{n}}+V_{\mathbf{n}}$ for all $\mathbf{n} \succeq \mathbf{1}$. By the Markov inequality, we have

$$
\begin{aligned}
& \sum_{\mathbf{n} \succeq \mathbf{1}} P\left(T_{\mathbf{n}} \geq \epsilon a_{\Gamma(\mathbf{n}+\mathbf{1})} \varphi_{2}^{(p-1) / p}(\Gamma(\mathbf{n}))\right) \\
& \quad \leq \frac{1}{2 \epsilon^{p}} \sum_{\mathbf{n} \succeq \mathbf{1}} \frac{1}{a_{\Gamma(\mathbf{n}+\mathbf{1})}^{p} \varphi_{2}^{p-1}(\Gamma(\mathbf{n}))} \mathbb{E} U_{\mathbf{n}}^{p}+\frac{1}{2 \epsilon} \sum_{\mathbf{n} \succeq \mathbf{1}} \frac{1}{a_{\Gamma(\mathbf{n}+\mathbf{1})}} \mathbb{E} V_{\mathbf{n}} .
\end{aligned}
$$

By Theorem 3.2, we have

$$
\begin{aligned}
& \sum_{\mathbf{n} \succeq \mathbf{1}} \frac{1}{a_{\Gamma(\mathbf{n}+\mathbf{1})}^{p} \varphi_{2}^{p-1}(\Gamma(\mathbf{n}))} \mathbb{E} U_{\mathbf{n}}^{p} \\
& \quad \leq C \sum_{\mathbf{n} \succeq \mathbf{1}} \frac{E\left\|U_{\mathbf{n}}\right\|^{p}}{a_{\mathbf{n}}^{p}} \leq C \sum_{\mathbf{n} \succeq \mathbf{1}} E\left\|\frac{Y_{\mathbf{n}}}{a_{\mathbf{n}}}\right\|^{p} \\
& \quad \leq C \sum_{\mathbf{n} \succeq \mathbf{1}} E\left\|\frac{Y_{\mathbf{n}}}{a_{\mathbf{n}}}\right\|^{\mu_{\mathbf{n}}} \leq C \sum_{\mathbf{n} \succeq \mathbf{1}} D_{\mathbf{n}} \frac{E \psi_{\mathbf{n}}\left(\left\|Y_{\mathbf{n}}\right\|\right)}{\psi_{\mathbf{n}}\left(a_{\mathbf{n}}\right)} \leq C \sum_{\mathbf{n} \succeq \mathbf{1}} A_{\mathbf{n}} \frac{E \psi_{\mathbf{n}}\left(\left\|X_{\mathbf{n}}\right\|\right)}{\psi_{\mathbf{n}}\left(a_{\mathbf{n}}\right)}
\end{aligned}
$$

Next, by Theorem 3.2,

$$
\begin{aligned}
& \sum_{\mathbf{n} \succeq \mathbf{1}} \frac{1}{a_{\Gamma(\mathbf{n}+\mathbf{1})}} \mathbb{E} V_{\mathbf{n}} \\
& \quad \leq C \sum_{\mathbf{n} \succeq \mathbf{1}} \frac{E\left\|V_{\mathbf{n}}\right\|}{a_{\mathbf{n}}} \leq C \sum_{\mathbf{n} \succeq \mathbf{1}} E\left\|\frac{Z_{\mathbf{n}}}{a_{\mathbf{n}}}\right\| \\
& \quad \leq C \sum_{\mathbf{n} \succeq \mathbf{1}} E\left\|\frac{Z_{\mathbf{n}}}{a_{\mathbf{n}}}\right\|^{\lambda_{\mathbf{n}}} \leq C \sum_{\mathbf{n} \succeq \mathbf{1}} \frac{1}{C_{\mathbf{n}}} \frac{E \psi_{\mathbf{n}}\left(\left\|Z_{\mathbf{n}}\right\|\right)}{\psi_{\mathbf{n}}\left(a_{\mathbf{n}}\right)} \leq C \sum_{\mathbf{n} \succeq \mathbf{1}} A_{\mathbf{n}} \frac{E \psi_{\mathbf{n}}\left(\left\|X_{\mathbf{n}}\right\|\right)}{\psi_{\mathbf{n}}\left(a_{\mathbf{n}}\right)} .
\end{aligned}
$$

Then (3.7), (3.8), and (3.9) yield (3.6).

Remark 3.5 The field of functions $\left\{\psi_{\mathbf{n}}, \mathbf{n} \succeq \mathbf{1}\right\}$ with $\psi_{\mathbf{n}}(x)=|x|^{p}$ satisfies the property (3.5).

Recall that the field of $\mathbb{E}$-valued random variables $\left\{X_{\mathbf{n}}, \mathbf{n} \in \mathbb{N}^{d}\right\}$ is said to be stochastically dominated by an $\mathbb{E}$-valued random variable $X$ if, for some $0<C<\infty$,

$$
P\left\{\left\|X_{\mathbf{n}}\right\| \geq x\right\} \leq C P\{\|X\| \geq x\}
$$

for all $\mathbf{n} \in \mathbb{N}^{d}$ and $x>0$.

Theorem 3.6 Let $\left\{X_{\mathbf{n}}, \mathcal{F}_{\mathbf{n}} ; \mathbf{n} \in \mathbb{N}^{d}\right\}$ be a blockwise- $\alpha$-strong* adapted field with respect to the blocks $\left\{\Delta_{\mathbf{k}}, \mathbf{k} \succeq \mathbf{1}\right\}$ in a real separable p-uniformly smooth Banach space $\mathbb{E}$ with $1<p \leq 2$. Let $\alpha_{1}, \ldots, \alpha_{d}$ be positive constants satisfying $\min \left\{\alpha_{1}, \ldots, \alpha_{d}\right\}=1$, let $q$ be the number of integers $s$ such that $\alpha_{s}=1=\min \left\{\alpha_{1}, \ldots, \alpha_{d}\right\}$. If $\left\{X_{\mathbf{n}} ; \mathbf{n} \in \mathbb{N}^{d}\right\}$ is stochastically dominated by an $\mathbb{E}$-valued random variable $X$. Then

$$
\sum_{\mathbf{n} \succeq \mathbf{1}} P\left(T_{n} \geq \epsilon\left|\Gamma^{\alpha}(\mathbf{n}+\mathbf{1})\right| \varphi_{2}^{(p-1) / p}(\Gamma(\mathbf{n}))\right)<C\left(E\|X\| \log _{+}^{q}\|X\|+1\right) .
$$


Proof For each $\mathbf{n} \succeq \mathbf{1}$, set

$$
\begin{aligned}
& Y_{\mathbf{n}}=X_{\mathbf{n}} I\left(\left\|X_{\mathbf{n}}\right\| \leq\left|\mathbf{n}^{\alpha}\right|\right), \quad Z_{\mathbf{n}}=X_{\mathbf{n}} I\left(\left\|X_{\mathbf{n}}\right\|>\left|\mathbf{n}^{\alpha}\right|\right), \\
& U_{\mathbf{n}}=\sup _{\mathbf{k} \in \Delta^{\mathbf{n}}}\left\|\sum_{\Gamma(\mathbf{n}) \leq \mathbf{i} \leq \mathbf{k}} Y_{\mathbf{i}}-E\left(Y_{\mathbf{i}} \mid \mathcal{F}_{\mathbf{i}-\alpha(\mathbf{i})}^{*}\right)\right\|, \\
& V_{\mathbf{n}}=\sup _{\mathbf{k} \in \Delta^{\mathbf{n}}}\left\|\sum_{\Gamma(\mathbf{n}) \leq \mathbf{i} \leq \mathbf{k}} Z_{\mathbf{i}}-E\left(Z_{\mathbf{i}} \mid \mathcal{F}_{\mathbf{i}-\alpha(\mathbf{i})}^{*}\right)\right\| .
\end{aligned}
$$

Since $\left\{X_{\mathbf{n}}, \mathcal{F}_{\mathbf{n}}, \mathbf{n} \succeq \mathbf{1}\right\}$ is a blockwise- $\alpha$-strong* adapted field with respect to the blocks $\left\{\Delta_{\mathbf{m}}, \mathbf{m} \succeq \mathbf{1}\right\}$ then it is clear that $\left\{Y_{\mathbf{n}}, \mathcal{F}_{\mathbf{n}}, \mathbf{n} \succeq \mathbf{1}\right\}$ and $\left\{Z_{\mathbf{n}}, \mathcal{F}_{\mathbf{n}}, \mathbf{n} \succeq \mathbf{1}\right\}$ are blockwise- $\alpha$-strong adapted fields with respect to the blocks $\left\{\Delta_{\mathbf{m}}, \mathbf{m} \succeq \mathbf{1}\right\}$. Moreover, for $\mathbf{n} \in \mathbb{N}^{d}$,

$$
X_{\mathbf{n}}+E\left(X_{\mathbf{n}} \mid \mathcal{F}_{\mathbf{n}-\alpha(\mathbf{n})}\right)=\left(Y_{\mathbf{n}}+E\left(Y_{\mathbf{n}} \mid \mathcal{F}_{\mathbf{n}-\alpha(\mathbf{n})}\right)\right)+\left(Z_{\mathbf{n}}+E\left(Z_{\mathbf{n}} \mid \mathcal{F}_{\mathbf{n}-\alpha(\mathbf{n})}\right)\right) .
$$

Then $T_{\mathbf{n}} \leq U_{\mathbf{n}}+V_{\mathbf{n}}$ for all $\mathbf{n} \succeq \mathbf{1}$. By the Markov inequality, Theorem 3.2, and Lemma 2.9, we have

$$
\begin{array}{rl}
\sum_{\mathbf{n} \geq \mathbf{1}} & P\left(T_{\mathbf{n}} \geq \epsilon|\Gamma(\mathbf{n}+\mathbf{1})| \varphi_{2}^{(p-1) / p}(\Gamma(\mathbf{n}))\right) \\
\leq & \frac{2^{p}}{\epsilon^{p}} \sum_{\mathbf{n} \geq \mathbf{1}} \frac{1}{|\Gamma(\mathbf{n}+\mathbf{1})|^{p} \varphi_{2}^{p-1}(\Gamma(\mathbf{n}))} \mathbb{E} U_{\mathbf{n}}^{p}+\frac{2}{\epsilon} \sum_{\mathbf{n} \geq \mathbf{1}} \frac{1}{|\Gamma(\mathbf{n}+\mathbf{1})|} \mathbb{E} V_{\mathbf{n}} \\
\leq & C \sum_{\mathbf{n} \geq \mathbf{1}} \frac{1}{\left|\mathbf{n}^{\alpha}\right|^{p}} E\left\|Y_{\mathbf{n}}\right\|^{p}+C \sum_{\mathbf{n} \geq \mathbf{1}} \frac{1}{\left|\mathbf{n}^{\alpha}\right|} E\left\|Z_{\mathbf{n}^{\alpha}}\right\| \\
\leq & C \sum_{\mathbf{n} \geq \mathbf{1}} \frac{1}{\left|\mathbf{n}^{\alpha}\right|^{p}} E\|X\|^{p} I_{\left\{\|X\|\left|\mathbf{n}^{\alpha}\right|\right\}}+C \sum_{\mathbf{n} \geq \mathbf{1}} \frac{1}{\left|\mathbf{n}^{\alpha}\right|} E\|X\| I_{\left\{\|X\| \leq\left|\mathbf{n}^{\alpha}\right|\right\}} \\
\leq & C \sum_{\mathbf{n} \geq \mathbf{1}} P\left\{\|X\| \geq\left|\mathbf{n}^{\alpha}\right|\right\}+C \sum_{\mathbf{n} \geq \mathbf{1}} \frac{1}{\left|\mathbf{n}^{\alpha}\right|} \int_{\left|\mathbf{n}^{\alpha}\right|}^{\infty} P\{\|X\| \geq t\} d t \\
& +C \sum_{\mathbf{n} \geq \mathbf{1}} \frac{1}{\left|\mathbf{n}^{\alpha}\right|^{p}} \int_{0}^{\left|\mathbf{n}^{\alpha}\right|^{p}} P\left\{\|X\|^{p} \geq t\right\} d t \\
\leq & C \sum_{\mathbf{n} \geq \mathbf{1}} \frac{1}{\left|\mathbf{n}^{\alpha}\right|} \int_{\left|\mathbf{n}^{\alpha}\right|}^{\infty} P\{\|X\| \geq t\} d t+C \sum_{\mathbf{n} \geq \mathbf{1}} \frac{1}{\left|\mathbf{n}^{\alpha}\right|^{p}} \int_{0}^{\left|\mathbf{n}^{\alpha}\right|^{p}} P\left\{\|X\|^{p} \geq t\right\} d t \\
\leq & C\left(E\|X\| \log _{+}^{q}\|X\|+1\right) .
\end{array}
$$

\section{Application to the strong law of large numbers}

By applying theorems in Section 3 we establish some results of strong laws of large numbers for fields of blockwise- $\alpha$-martingale differences with values in a $p$-uniformly smooth Banach space.

In the rest of this paper, we denote by $\left\{X_{\mathbf{n}}, \mathcal{F}_{\mathbf{n}}: \mathbf{n} \succeq \mathbf{1}\right\}$ the blockwise- $\alpha$-martingale difference field with respect to the blocks $\left\{\Delta_{\mathbf{k}}, \mathbf{k} \succeq \mathbf{1}\right\}$. When $\alpha(\mathbf{k})=\mathbf{M}$ for all $\mathbf{k}$, it is called a strong blockwise- $M$-martingale difference field, and we set

$$
S_{\mathbf{k}}=\sum_{\mathbf{1} \leq \mathbf{i} \leq \mathbf{k}} X_{\mathbf{i}}
$$


Let $\left\{a_{\mathbf{n}}, \mathbf{n} \succeq \mathbf{1}\right\}$ be a nondecreasing field of positive constants such that

$$
1<\liminf _{\mathbf{n} \prec \mathbf{m} \leq \mathbf{n}+\mathbf{1}} \frac{a_{\Gamma(\mathbf{m})}}{a_{\Gamma(\mathbf{n})}} \leq \limsup _{\mathbf{n} \prec \mathbf{m} \leq \mathbf{n}+\mathbf{1}} \frac{a_{\Gamma(\mathbf{m})}}{a_{\Gamma(\mathbf{n})}}<\infty .
$$

Theorem 4.1 Let $1 \leq p \leq 2$, and let $\mathbb{E}$ be a separable Banach space, then the following three statements are equivalent:

(i) $\mathbb{E}$ is p-uniformly smooth.

(ii) $\left\{X_{\mathbf{n}}, \mathcal{F}_{\mathbf{n}}, \mathbf{n} \succeq \mathbf{1}\right\}$ is a blockwise- $\alpha$-martingale difference field in $\mathbb{E}$ with respect to the blocks $\left\{\Delta_{\mathbf{k}}, \mathbf{k} \succeq \mathbf{1}\right\},\left\{a_{\mathbf{n}}, \mathbf{n} \succeq \mathbf{1}\right\}$ is a nondecreasing field of positive constants satisfying (4.1). If

$$
\sum_{\mathbf{n} \succeq \mathbf{1}} \frac{1}{a_{\mathbf{n}}} E\left\|X_{\mathbf{n}}\right\|^{p}<\infty,
$$

then we have

$$
\frac{1}{a_{\mathbf{n}} \phi_{2}^{(p-1) / p}(\mathbf{n})} \max _{\mathbf{1} \leq \mathbf{k} \leq \mathbf{n}}\left\|S_{\mathbf{k}}\right\| \rightarrow 0 \quad \text { a.s. as }|\mathbf{n}| \rightarrow 0 .
$$

(iii) $\left\{X_{\mathbf{n}}, \mathcal{F}_{\mathbf{n}}: \mathbf{n} \succeq \mathbf{1}\right\}$ is a blockwise-M-martingale difference field in $\mathbb{E}$ with respect to the blocks $\left\{\Delta_{\mathbf{k}}, \mathbf{k} \succeq \mathbf{1}\right\},\left\{a_{\mathbf{n}}, \mathbf{n} \succeq \mathbf{1}\right\}$ is a nondecreasing sequence of positive constants satisfying (4.1). If (4.2) holds, then we have

$$
\frac{1}{a_{\mathbf{n}}\left(\phi_{1}(\mathbf{n})\right)^{(p-1) / p}} \max _{\mathbf{1} \leq \mathbf{k} \leq \mathbf{n}}\left\|S_{\mathbf{k}}\right\| \rightarrow 0 \quad \text { a.s. as }|\mathbf{n}| \rightarrow 0 .
$$

Proof ((i) $\Rightarrow$ (ii)) By (4.2) and Theorem 3.2, we have

$$
\frac{T_{\mathbf{n}}}{a_{\Gamma(\mathbf{n}+\mathbf{1})} \phi_{2}(\mathbf{n})} \rightarrow 0 \quad \text { a.s. }
$$

For $\mathbf{n} \succeq \mathbf{1}$, let $\mathbf{m} \succeq \mathbf{0}$ be such that $\mathbf{n} \in \Delta^{(\mathbf{m})}$, by Lemma 2.9, we have

$$
\begin{aligned}
0 & \leq \frac{1}{a_{\mathbf{n}}\left(\phi_{2}(\mathbf{n})\right)^{(p-1) / p}} \sup _{\mathbf{k} \leq \mathbf{n}}\left\|\sum_{\mathbf{1} \leq \mathbf{i} \leq \mathbf{k}} X_{\mathbf{i}}\right\| \\
& \leq \frac{1}{a_{\Gamma(\mathbf{m})}} \sup _{\mathbf{k} \leq \mathbf{m}}\left\|\sum_{\mathbf{1} \leq \mathbf{i} \leq \mathbf{k}} a_{\Gamma(\mathbf{i}+1)} \frac{T_{\mathbf{i}}}{a_{\Gamma(\mathbf{i}+\mathbf{1})} \phi_{2}(\Gamma(\mathbf{i}))}\right\| \rightarrow 0 \quad \text { a.s. }
\end{aligned}
$$

((ii) $\Rightarrow$ (iii)) When $\alpha(\mathbf{k})=\mathbf{M}$ for all $\mathbf{k} \succeq \mathbf{1}$, with a note that $\varphi_{2}(\mathbf{m})=|\mathbf{M}| \varphi_{1}(\mathbf{m})$ for all $\mathbf{m} \succeq \mathbf{1}$. We have (4.3).

((iii) $\Rightarrow$ (i)) Assume that (iii) holds. Let $\left\{X_{n}, \mathcal{G}_{n}, n \geq 1\right\}$ be a martingale difference sequence in $\mathbb{E}$ such that

$$
\sum_{n=1}^{\infty} \frac{E\left\|X_{n}\right\|^{p}}{n^{p}}<\infty
$$

For $\mathbf{n} \succeq \mathbf{1}$, put $X_{\mathbf{n}}=X_{n_{1}}$ if $n_{i}=1(2 \leq i \leq d)$ and $X_{\mathbf{n}}=0$ if there exists a positive integer $i$ $(2 \leq i \leq d)$ such that $n_{i} \geq 2$,

$$
\mathcal{F}_{\mathbf{n}}=\mathcal{G}_{n_{1}} \quad \text { for all } \mathbf{n}=\left(n_{1}, \ldots, n_{d}\right) \in \mathbb{N}^{d} .
$$


Then $\left\{X_{\mathbf{n}}, \mathcal{F}_{\mathbf{n}}, \mathbf{n} \succeq \mathbf{1}\right\}$ is a blockwise-1-martingale difference field with respect to the blocks $\prod_{k=1}^{d}\left[2^{k}, 2^{k+1}\right)$ in $\mathbb{E}$ and $\sum_{\mathbf{n} \succeq \mathbf{1}}^{\infty} \frac{E\left\|X_{\mathbf{n}}\right\|^{p}}{|\mathbf{n}|^{p}}=\sum_{n=1}^{\infty} \frac{E\left\|X_{n}\right\|^{p}}{n^{p}}<\infty$. Let $a_{\mathbf{n}}=|\mathbf{n}|$ for all $\mathbf{n} \succeq \mathbf{1}$. Then (4.1) and (4.2) hold. Thus, by (iii),

$$
\lim _{|\mathbf{n}| \rightarrow \infty} \frac{1}{|\mathbf{n}|\left(\phi_{1}(\mathbf{n})\right)^{(p-1) / p}} \sum_{\mathbf{1} \leq \mathbf{i} \leq \mathbf{n}} X_{\mathbf{i}}=0 \quad \text { a.s. }
$$

Note that $\phi_{1}(\mathbf{n})=1, \mathbf{n} \succeq \mathbf{1}$, and we have

$$
\lim _{|\mathbf{n}| \rightarrow \infty} \frac{1}{|\mathbf{n}|} \sum_{\mathbf{1} \leq \mathbf{i} \leq \mathbf{n}} X_{\mathbf{i}}=0 \quad \text { a.s. }
$$

Taking $n_{i}=1$ for all $2 \leq i \leq d$ and letting $n_{i} \rightarrow \infty$, we obtain

$$
\lim _{n_{1} \rightarrow \infty} \frac{1}{n_{1}} \sum_{i=1}^{n_{1}} X_{i}=0 \quad \text { a.s. }
$$

Then by Theorem 2.2 of Hoffmann-Jørgensen and Pisier [11], $\mathbb{E}$ is $p$-uniformly smooth.

Remark 4.2 In Theorem 4.1, when $d=2, \alpha(\mathbf{n})=\mathbf{1}, \Delta_{\mathbf{k}}=\prod_{k=1}^{d}\left[2^{k}, 2^{k+1}\right)$ we have the result in Theorem 3.2 in [3]. When $d=2, \alpha(\mathbf{n})=\mathbf{M}, \mathbb{E}=\mathbb{R}, \Delta_{\mathbf{k}}=\prod_{k=1}^{d}\left[2^{k}, 2^{k+1}\right),\left\{X_{\mathbf{n}} ; \mathbf{n} \succeq \mathbf{1}\right\}$ is a double of mean zero random variables and we have a part of Theorem 3.1 in [6].

$\left\{X_{\mathbf{n}}, \mathcal{F}_{\mathbf{n}} ; \mathbf{n} \succeq \mathbf{1}\right\}$ is said to be a strong* blockwise- $\alpha$-martingale difference field if it is a blockwise- $\alpha$-strong* adapted field as well as a blockwise- $\alpha$-martingale difference field.

Theorem 4.3 Let $1 \leq p \leq 2$, and let $\mathbb{E}$ be a separable Banach space, then the following two statements are equivalent:

(i) $\mathbb{E}$ is p-uniformly smooth.

(ii) $\left\{X_{\mathbf{n}}, \mathcal{F}_{\mathbf{n}}, \mathbf{n} \succeq \mathbf{1}\right\}$ is a strong* blockwise- $\alpha$-martingale difference field in $\mathbb{E}$ with respect to the blocks $\left\{\Delta_{\mathbf{k}}, \mathbf{k} \succeq \mathbf{1}\right\},\left\{\psi_{\mathbf{n}}, \mathbf{n} \geq \mathbf{1}\right\}$ is a field of positive Borel functions satisfying (3.5). If

$$
\sum_{\mathbf{n} \succeq \mathbf{1}} A_{\mathbf{n}} \frac{E \psi_{\mathbf{n}}\left(\left\|X_{\mathbf{n}}\right\|\right)}{\psi_{\mathbf{n}}\left(a_{\mathbf{n}}\right)}<\infty,
$$

where $A_{\mathbf{n}}=\max \left\{\frac{1}{C_{\mathbf{n}}}, D_{\mathbf{n}}\right\}$, then we have (4.3).

Proof $((\mathrm{i}) \Rightarrow$ (ii)) By Theorem 3.4 and by the same argument as in the proof of Theorem 4.1.

((ii) $\Rightarrow$ (i)) Assume that (ii) holds. Let $\left\{X_{n}, \mathcal{G}_{n}, n \geq 1\right\}$ be a martingale difference sequence in $\mathbb{E}$ such that

$$
\sum_{n=1}^{\infty} \frac{E\left\|X_{n}\right\|^{p}}{n^{p}}<\infty
$$

For $\mathbf{n} \succeq \mathbf{1}$, put $X_{\mathbf{n}}=X_{n_{1}}$ if $n_{i}=1(2 \leq i \leq d)$ and $X_{\mathbf{n}}=0$ if there exists a positive integer $i$ $(2 \leq i \leq d)$ such that $n_{i} \geq 2$,

$$
\mathcal{F}_{\mathbf{n}}=\mathcal{G}_{n_{1}} \quad \text { for all } \mathbf{n}=\left(n_{1}, \ldots, n_{d}\right) \in \mathbb{N}^{d}
$$


Then $\left\{X_{\mathbf{n}}, \mathcal{F}_{\mathbf{n}}, \mathbf{n} \succeq \mathbf{1}\right\}$ is a blockwise-1-martingale difference and strong* adapted field with respect to the blocks $\prod_{k=1}^{d}\left[2^{k}, 2^{k+1}\right)$ in $\mathbb{E}$ and we have $\sum_{\mathbf{n} \geq \mathbf{1}}^{\infty} \frac{E\left\|X_{n}\right\|^{p}}{\left.\mathbf{n}\right|^{p}}=\sum_{n=1}^{\infty} \frac{E\left\|X_{n}\right\|^{p}}{n^{p}}<\infty$. Put $\psi_{\mathbf{n}}(x)=x^{p}, \lambda_{\mathbf{n}}=1, \mu_{\mathbf{n}}=p, C_{\mathbf{n}}=1, D_{\mathbf{n}}=1, \mathbf{n} \succeq \mathbf{1}$ and $a_{\mathbf{n}}=|\mathbf{n}|$ for all $\mathbf{n} \succeq \mathbf{1}$. Thus, by (ii) and by the same argument as in the proof of Theorem 4.1, we have (i).

Theorem 4.4 Let $\left\{X_{\mathbf{n}}, \mathcal{F}_{\mathbf{n}} ; \mathbf{n} \succeq \mathbf{1}\right\}$ be a blockwise- $\alpha$-strong* adapted field with respect to the blocks $\left\{\Delta_{\mathbf{k}}, \mathbf{k} \succeq \mathbf{1}\right\}$ in a real separable p-uniformly smooth Banach space $\mathbb{E}$ with $1<p \leq 2$. Let $\alpha_{1}, \ldots, \alpha_{d}$ be positive constants satisfying $\min \left\{\alpha_{1}, \ldots, \alpha_{d}\right\}=1$, let $q$ be the number of integers s such that $\alpha_{s}=1=\min \left\{\alpha_{1}, \ldots, \alpha_{d}\right\}$. If $\left\{X_{\mathbf{n}} ; \mathbf{n} \in \mathbb{N}^{d}\right\}$ is stochastically dominated by an $\mathbb{E}$-valued random variable $X$ such that $E\left(\|X\| \log _{+}^{q}\|X\|\right)<\infty$. Then

$$
\frac{1}{\left|\mathbf{n}^{\alpha}\right| \phi_{2}(\mathbf{n})} \max _{\mathbf{1} \leq \mathbf{k} \leq \mathbf{n}}\left\|S_{\mathbf{k}}\right\| \rightarrow 0 \quad \text { a.s. as }|\mathbf{n}| \rightarrow 0 .
$$

Proof Using Theorem 4.3 and by the same argument as in the proof of Theorem 4.1, we have (4.5).

Remark 4.5 In Theorem 4.3, when $d=2, \alpha(\mathbf{n})=1, \Delta_{\mathbf{k}}=\Delta^{\mathbf{k}}=\prod_{k=1}^{d}\left[2^{k}, 2^{k+1}\right)$ we have the result in Theorem 3.2(ii) in [7].

\section{Competing interests}

The authors declare that there is no conflict of interests regarding the publication of this article.

\section{Authors' contributions}

All authors contributed equally and significantly in writing this article. All authors read and approved the final manuscript.

\section{Acknowledgements}

The authors would like to express their gratitude to the referees for their detailed comments and valuable suggestions, which helped them to improve the manuscript. The research has been supported by VNU Grant No. QG.13.02.

\section{Received: 2 June 2014 Accepted: 23 October 2014 Published: 04 Nov 2014}

\section{References}

1. Móricz, F: Strong limit theorems for block-wise $m$-dependent and block-wise quasiorthogonal sequences of random variables. Proc. Am. Math. Soc. 101, 709-715 (1987)

2. Gaposhkin, VF: On the strong law of large numbers for block-wise independent and block-wise orthogonal random variables. Theory Probab. Appl. 39, 667-684 (1995)

3. Huan, NV, Quang, NV, Volodin, A: Strong laws for blockwise martingale difference arrays in Banach spaces. Lobachevskii J. Math. 31(4), 326-335 (2010)

4. Móricz, F, Stadtmüller, U, Thalmaier, M: Strong laws for block-wise $\mathcal{M}$-dependent random fields. J. Theor. Probab. 21, 660-671 (2008)

5. Stadtmüller, U, Thalmaier, M: Strong laws for delayed sums of random fields. Acta Sci. Math. 75(3-4), 723-737 (2009)

6. Stadtmüller, U, Thanh, LV: On the strong limit theorems for double arrays of block-wise M-dependent random variables. Acta Math. Sin. Engl. Ser. 27, 1923-1934 (2011)

7. Quang, NV, Huan, NV: On the strong law of large numbers and $\mathcal{L}_{p}$-convergence for double arrays of random elements in p-uniformly smooth Banach spaces. Stat. Probab. Lett. 79, 1891-1899 (2009)

8. Huan, NV, Quang, NV: The Doob inequality and strong law of large numbers for multidimensional arrays in general Banach spaces. Kybernetika 48(2), 254-267 (2012)

9. Castaing, C, Quang, NV, Thuan, NT: A new family of convex weekly compact valued random variables in Banach space and applications to laws of large numbers. Stat. Probab. Lett. 82(1), 84-95 (2012)

10. Gut, A: Convergence rates in the central limit theorem for multidimensionally indexed random variables. Studia Sci. Math. Hung. 37, 401-418 (2001)

11. Hoffmann-Jørgensen, J, Pisier, G: The law of large numbers and the central limit theorem in Banach spaces. Ann. Probab. 4(4), 587-599 (1976) 